\title{
Acquisition Order in Negative Prefixes and Negative Adverbs for Chinese Learners : A Corpus-Based Approach
}

\author{
Xinyue Shui ${ }^{1, *}$, Xiaoxi Chen" ${ }^{2 *}$, Yongfeng Qiu³ ${ }^{3}$ Yuwei Yan ${ }^{4}$ \\ ${ }^{1}$ Rossier School of Education, University of Southern California, Los Angeles, USA, xinyueshui05@gmail.com \\ ${ }^{2}$ Chongqing University, Chongqing, China \\ ${ }^{3}$ Guangdong University of Finance\&Economics, Guangzhou, China \\ ${ }^{4}$ International Department, Chengdu No.7 High School, Chengdu, China \\ * They are both first authors.
}

\begin{abstract}
In English, negation includes explicit negation such as negations with negative suffixes and implicit negation like negative adverbs. In this study, we focus on negative prefixes and negative adverbs which are two difficulties for Chinese learners. And we tried to find out: a) learners' acquisition features and acquisition order of negative prefixes and adverbs, and b) whether natural order exists in acquiring negative prefixes and adverbs. Results showed that with the increase of educational level, learners acquire more negative prefixes and adverbs, and Chinese learners share similar using preferences with native speakers.
\end{abstract}

Keywords: SLA, corpus-based study, Error analysis theory, Language transfer theory

\section{INTRODUCTION}

Negation can be divided into two types: explicit negation and implicit negation. Explicit negation refers to users use no, not, or negative affixes such as un- or imwhile implicit negation refers to negative sentences without these words or affixes, for example, using negative adverbs. This study focuses on negative prefixes and negative adverbs which are two difficulties for Chinese learners.

For Chinese learners and English teachers, using "no" or "not" is not a difficult thing because Chinese has a similar structure; the positive transfer would help students acquire them quickly. However, for Chinese learners, words with negative suffixes are not easily acquired. As for negative adverbs, Chinese learners also feel difficulty about them because, in Chinese, even adverbs with low frequency are not considered as negative words.

\section{LITERATURE REVIEW}

Linguists categorize negations into explicit and implicit negation [1]. Explicit negation employs explicit negative particles such as not, negative affixes such as prefix dis-, or suffix -less; or negative adverbs, or determiners.
Similarly, studies have also found that the acquisition order of one's second language (L2) shares certain similarities with that of one's first language (L1) [2]. Second language competence is developed following a predictable series of benchmarks [3]. Morpheme order studies are part of the theoretical foundation for the Natural Order Hypothesis (NOH) [4].

To verify the existence of acquisition order, positive and negative transfer, the error analysis, misspelling categorization may be helpful.

Positive transfer occurs when a L1 structure or rule is applicable and proper in L2, which is also the easiest for learners; whereas negative transfer (or interference) occurs when an L1 structure or rule is incorrect or improper in L2 utterance [5].

Similarities in meaning and distribution between English and Chinese negative prefixes may make the positive transfer available. The affixes in modern Chinese normally are derivative ones [6]. Modern Chinese Dictionary [7] contains 29 affixes, of which 7 prefixes include "非". There are 36 affixes listed in the Syllabus of Grammatical Grades of Standard Chinese Language [8], including the prefixes "无" and "非".

Negative adverbs "无" and "非" are also prefixes in 
Chinese and both mean "no", and are explicit negative indicators. Similarly, English word formation abounds explicit negative prefixes like dis- and its morpheme variations including im-, il-, ir-. However, many negative prefixes in English can be translated into "不" in Chinese like unhappy (不高兴). Here “不” is not categorized as a prefix but as an independent negative character, and “不 高兴" is classified as a phrase [6]. Nevertheless, the positive transfer theory can probe the possible similarities in acquisition orders in negative prefixes and negative adverbs between English native speakers and Chinese learners.

Another transfer is negative transfer and so-called, interference. Negative transfer can be classified into 3 categories. The first one is "underproduction" [9], caused by unfamiliarity with the target language and then learners may use less frequent words. The second category is "overproduction" that learners may repeatedly use one or two familiar words even inappropriately [9]. "Mis-interpretation" is the third category of negative transfer which occurs when students mistakenly translate their L1 into L2 [10].

Negative transfer is one of the causes of errors [5]. Unlike mistakes or lapses, errors are signs that learners have not fully mastered "some institutionalized language system" [11]. The modern definition of the error is influenced by contrastive study. For contrastive study supporters, language users' cognitive structures are acquired by acquiring the data-processing and hypothesis formation process [12] and errors demonstrate this process. Then, Corder proposed Error Analysis. Errors represent how learners reconstruct prior knowledge of L2 during learning [11] for both L1 and L2 learners. Error analysis allows us to understand "the inner working language learning process" [13].

Richards [14] also classified 3 sources of errors: a) interference error, which is influenced by mother tongues; b) intralingual error, which is caused by incorrect generalization within L2; c) developmental error, which reflects learners make hypotheses based on their limited knowledge about L1. Overgeneralization, belonging to intralingual error, occurs when learners use a deviant structure, which is common for students who do not fully acquire. Overuse is another common error belonging to developmental error which may brought by insufficient vocabulary and learners' L1.

Spelling errors are also common in SLA process. Wang \& Sun [15] categorizes the common misspellings by Chinese learners into a) phonological deviation means the misspelled item changes the pronunciation, b) graphemic deviation does not change the pronunciation of its correct version but the formation and c) Morphological deviations are incorrect in word formation. Such deviations may be explained by productivity because the frequency of each prefix may influence learners' familiarity to it. The misspelling analysis also demonstrates that learners' sensitivity to phonological awareness in phoneme positively contributes to their performance in spelling [16].

\section{REASEARCH QUESTION AND METHODOLOGY}

There have been several corpus-based studies focusing on negation. However, the corpora they used are not up-to-date enough. Most researchers also mainly used translation task to study, which is not authentic.

Based on the abovementioned gaps, we compare the usage of common negative prefixes and common negative adverbs in TECCL and BNC (except the spoken sub-corpus) to answer the following questions:

a. Compared to native speakers, what are the features of acquisition in the usage of negative adverbs and negative prefixes by Chinese English learners?

b. Is there a relationship between the acquisition order and the educational levels of Chinese learners? What are the acquisition order of negative adverbs and negative prefixes for these learners?

c. How does Chinese influence learners' English acquisition in negative adverbs and negative prefixes?

TECCL was constructed and completed in 2015 which has collected compositions (1.82 million words) from Chinese English learners in primary schools, middle schools and universities. Among them, the primary school section contains 6,424 words while the primary school and university section contain 429,091 and $1,530,408$ words respectively [17]. The reference corpus used in this paper is the British National Corpus (BNC), This monolingual corpus has collected about 100million-word texts and divided them into sections like spoken language or magazines [18]. In this research, we only selected the written English sections (86,299,736 words in total) to make comparisons as TECCL is a written English corpus.

We randomly chose negative suffixes and selected the most common used negative words (including its derivative) in every randomly extracted suffix from BNC. And then we randomly chose the most common negative adverbs in BNC.

Then we searched for word occurrences of words from BNC in TECCL. Finally, by comparing the lexical usage of the same affix between Chinese learners and native English speakers, we studied the differences between Chinese learners and native English speakers in the use of negative affixes and whether Chinese learners' use of negative words will be affected by their education level.

All data were normalized by using the formula: 


\section{Frequency $/ \mathrm{mil}=\left(\frac{\text { total matches }}{\text { the number of words in sub-corpus }}\right) *$ 1, 000,000}

In the subsequent analysis of the results, we stratified TECCL data according to education level, and then compared it with the native speakers with high educational level to study whether the choice of negative words is affected by educational background. Last, we integrated all data to make a chart of the total frequency of suffixes of BNC and TECCL to make a comparison and discuss which negative affix do Chinese learners master best

\section{RESULTS}

The frequencies of use of these negative prefixes in TECCL and BNC were similar except for the prefixes dis- and un- which had a great difference in the two corpora. Figure 1 shows standardized data that Chinese English learners use dis- in the highest frequency among 11 prefixes and there were 62.3 words with un- in every million words, which means Chinese English learners prefer to use dis- most to any other prefixes.

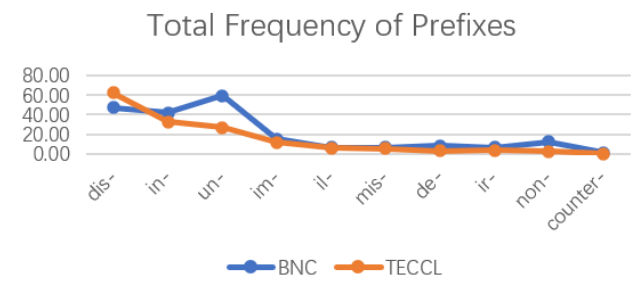

Figure 1. Total Frequency (per mil) of Prefixes

Figure 2 shows the frequency of 10 words with imnegative prefixes in TECCL in the order of education level from low to high. We could see that with the exception of the word "impossible", which saw a rapid rise in frequency from 0 in primary to 64.04 words in university, the use of the rest of the words basically rose slowly from primary school to college with similar upward trends (no more than 14).

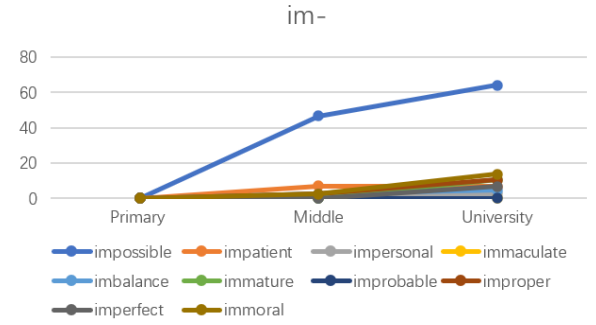

Figure 2. Total Frequency (per mil) of im- in TECCL

But there are three prefixes are not preferred by Chinese learners, even for Chinese university learners: il-, non-, and counter-. Here we take il- as an example.

Figure 3 shows the frequency of learners from different educational level using il- in their compositions. Although the line of "illegal" rose dramatically, from 0 in primary school to just above 50 words per million in university, the frequency of other words with il- in every million words remained around 0 , meaning that even learners' English proficiency level becomes higher, they are not likely to form words with the prefix il-.

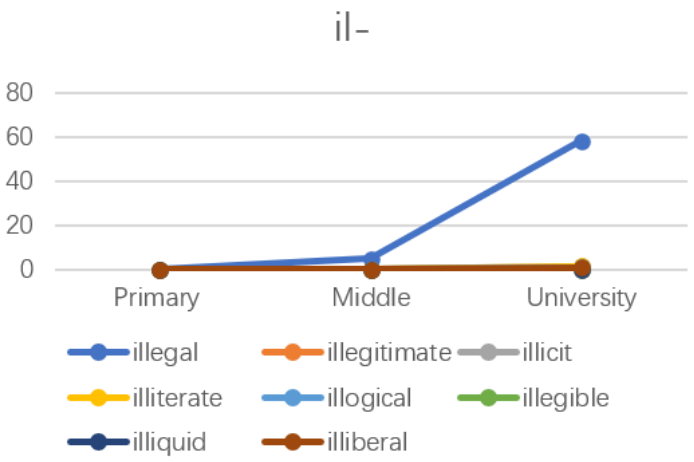

Figure 3. Frequency of il- used by learners from different educational level

Other negative affixes saw a similar pattern: the word frequency of over half of the words with detected negative affixes increased from a lower education level to higher education level. The frequency statistics of de-, non-, counter-, dis-, in-, un-, ir- and mis- affixes in TECCL under 3 different educational levels can be seen in Appendix A.

The five adverbs were also similar to the negative prefix. Figure 4 shows that in addition to the rapid increase in the frequency of use of "hardly" from primary school to middle school to 79.24 , and a slight decrease to 69.93 from middle school to college, all other adverbs are acquired more with the increase of educational level.

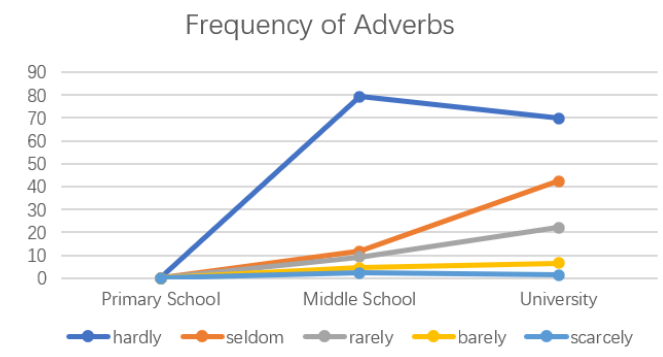

Figure 4. Total Frequency (per mil) of adverbs in TECCL

We compared 10 most common words with "im-" in BNC and in TECCL and concluded that the frequency of use of the 10 words with the im- was similar in both corpora, with the difference between the two being no more than 17.66 in every million words. 


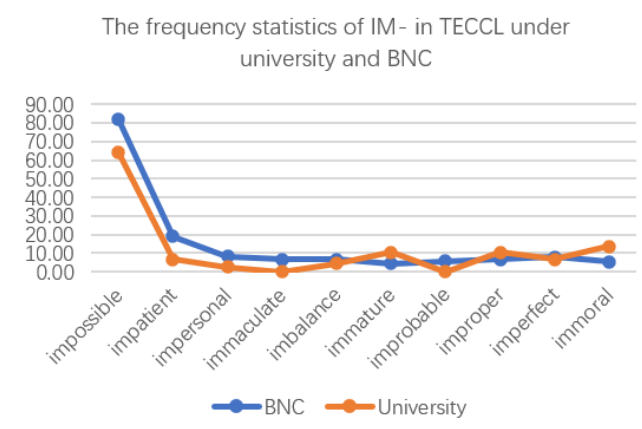

Figure 5. Total Frequency (per mil) of im- in BNC and in TECCL university-sub-corpus

All of the remaining 9 negative affixes saw a similar pattern: the word frequency of all of 10 words with detected negative affixes was similar between the use situation in BNC and in TECCL.

The five adverbs saw a similar characteristic with the negative prefixes. By observing the word frequency of these five frequently used negative adverbs after standardization in TECCL under university level and in $\mathrm{BNC}$, we could convert these data into two lines in one graph. Figure 6 shows that the word frequency in TECCL under university level and $\mathrm{BNC}$ is similar within the difference about 27 words in every million words.

The frequency of negative adverbs in TECCL (university) and BNC

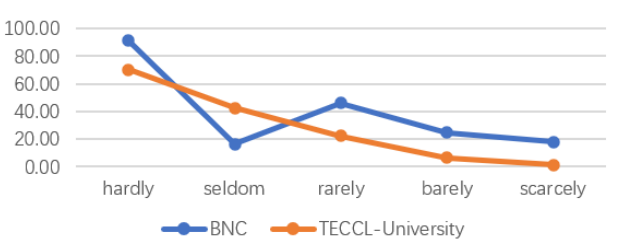

Figure 6. The comparison between adverbs in BNC and in TECCL university-sub-corpus

\section{DISCUSSION}

As noted in the literature review, "无" and "非" can both serve as explicit negative prefixes, both meaning "no". Likewise, explicit negative prefixes, such as dis-, counter-, in-, im-, il-, and ir- also abound in English word-formation. However, although “不” is not categorized as a prefix but as an independent negative character [6], many of these negative prefixes in English are expressed by "不" in Chinese. “不” serves as the grammaticalized prefix in word-formation of Chinese negations.

Combined with the know-how from the positive transfer theory, this explains why Chinese learners can understand and acquire most English explicit negative words in a quite natural way. Chinese negative suffixes share similar semantic and pragmatic functions as English negative prefixes including dis-, in-, im-, il-, ir-, non-, and counter-. Such similarities show the similar acquisition orders of negation affixes between Chinese learners and English native speakers.

Chinese learners and native speakers have a noticeable acquisition gap in negative prefixes. In TECCL, spelling errors occur in negative words. Although the sample amount seems quite negligible, we found spelling errors concentrate on in- and its morpheme variation im-, as well as on un-.

We reckon the major two reasons behind misspellings are a) blurry awareness of phonological accuracy, and b) overgeneralization. Students' sensitivity to phonological awareness in phoneme positively contributes to their performance in spelling. The misspelling of "inpersonal" and "inpossible" belongs to phonological deviations [15] in which the pronunciation of the correct versions are changed by inserting other letters. The reason behind the occurrence of such mistakes can usually be attributed to phonological accuracy between "in" and "im".

On the other hand, we clarify the misspelling of "unmoral" and "unbalance" as morphological deviations [15], with both "moral" and "balance" being wrongly combined with the incorrect choice of "un" as their prefix. The reason is overgeneralization. For speakers, it is reasonable to put the more common prefix "un-" before the stems "moral" and "balance" to form derivatives. From the perspective of productivity, un- is widely acknowledged as far more productive than in- [19]. This may explain why an over-generalized preference manifests in the spellers when they choose un- as the prefix.

The adverb is another category studied in the research. According to Zhang [20], adverbs in written Chinese appear along with verbs, adjectives and nouns; in short, it can only be adverbial. However, adverbs in English can both modify verbs, adjectives, nouns and even a whole sentence, and can also be used alone.

To answer the first question, we should focus on similarities and differences between negative adverb usage in TECCL and BNC. The similarity and difference lying in definitions of adverbs in Chinese and English can be the reason why we got the general tendencies of common adverbs in two corpora. In modern Mandarin, the negative adverb “不” is added before verbs to express negation or unwillingness, such as

我不想学习。

I don't want to study.

While another negative adverb “没” is used to describe a negation to a past facts like

昨天我没去。

I didn't go yesterday.

As Chinese speakers use adverbs to modify other words, Chinese learners keep an awareness of using 
adverbs in appropriate registers to express meaning more accurately. However, the general tendency shows that frequencies of all adverbs except "seldom" used in TECCL are almost half of those in BNC. One possible reason is that it is common for native speakers to use adverbs as a short sentence, such as using "hardly" to answer how often questions. The meaning of this word is “not often" while in Chinese we would like to say “不常” (adverbs +adjective). Another reason is that although frequency adverbs in Chinese are divided into 5 levels [21], the lowest frequency adverbs like “偶尔” (occasionally) are not considered as a negative word. As a result, when Chinese learners use English, they tend to use the "not +adverb" structure priorly.

The acquisition order within negative adverbs selected is "hardly, seldom, rarely, barely, and then scarcely". The general tendency based on educational level demonstrates the natural order within L2 learners. The results show that with the increase of grade, almost all lines go up except the line of "hardly" show a slight decline. In addition, when students receive higher education, they become older. This is the reason why we believe the result demonstrates that there is a relationship between age and acquisition order.

Although we have found the acquisition order, the reason why "hardly" is used more in middle school than in university is worth discussing. This might also because of the "overuse". We can conclude that for learners at this age, "hardly" is almost the only negative adverbs they have fully mastered. However, when they get older and receive higher education, the frequency of other words gets higher, and then the frequency of "hardly" decreases a little.

The third question can be answered by discussing "seldom". As mentioned above, seldom is an exception in comparison between the general tendency of TECCL and of BNC. To make insight into this exception, the negative transfer from L1 should be noted. In Chinese, many negative adverbs like "hardly" and "seldom" can be expressed in “不常”. To Chinese learners, they are almost the same; not many of them can distinguish these synonyms. However, for native speakers, "seldom" is formal, and it cannot be used in every register. With the influence of L1, Chinese learners overuse it without much consideration.

\section{CONCLUSION}

In this corpus-based study, we compared the frequency of negative adverbs and suffixes in BNC and TECCL and discussed whether the acquisition order of two suffixes and adverbs exists in Chinese learners, what are differences of usages between English native speakers and Chinese learners. We have gotten the conclusion that there is an order and for Chinese learners, L1 both negatively and positively influences their
English acquisition, but sometimes overuse and overgeneralization make their language usage less "authentic" and "native".

\section{REFERENCES}

[1] Jespersen, Negation in English and other languages. AF: Høst, 1917.

[2] H.C. Dulay and M.K. Burt, "Should We Teach Children Syntax?". Journal of Language Learning, Language Learning, vol.23, 1973, pp.245-258.

[3]J.G.D. Villiers and P.A.D. Villiers, “A cross-sectional study of the acquisition of grammatical morphemes in child speech". Journal of psycholinguistic research, vol.3,1973, pp. 267-278.

[4] S.D. Krashen, "Inquiries \& insights: second language teaching: immersion \& bilingual education, literacy". Alemany Press, 1985.

[5] R. Lado, Linguistics across Cultures. Ann Arbor: University of Michigan Press, 1957.

[6] X. Lu, Chinese grammar analysis. Beijing: Commercial Press, 1979.

[7] Dictionary Editing Office, Institute of language, Chinese Academy of Social Sciences. Modern Chinese Dictionary (6th Edition). Beijing: Commercial Press, 2012.

[8] Department of Chinese proficiency test, office of the national leading group for teaching Chinese as a foreign language. Syllabus of Chinese proficiency standard grammar. Beijing: Higher Education Press, 1998.

[9] W. Dai, \& D. Wang, "Language transfer studies: issues and reflections". Journal of Foreign Languages, vol.2, 2002, pp. 1-9.

[10] T. Odlin, Language transfer. New York: Cambridge University Press, 1989.

[11] S. P. Corder, "Error analysis, interlanguage and second language acquisition". Language Teaching, vol.4, 1975, pp.201-218

[12] H. Dulay, \& M. Burt, "Natural sequences in child second language acquisition". Language Learning, vol.24, 1974, pp.37-53.

[13] R. Ellis, Understanding second language acquisition. Oxford: Pergamon Institute of English, 1985.

[14] J. C. Richards, J. Platt, \& H. Platt, Longman Dictionary of Language Teaching and Applied Linguistics. London: Longman, 1992.

[15] X. Wang, L. Sun, "Re-analysis of Chinese students' English spelling errors". Foreign language teaching and research, vol.4, 2004, pp.299-304.

[16] C. Chen, "How does the mismatches in English spelling come into being?". Modern foreign languages, vol. 4, 1983, pp.34-37.

[17] X. Xue, "Ten-thousand English Compositions of Chinese Learners (The TECCL corpus), Version 1.1". The National Research Centre for Foreign 
Language Education, Beijing Foreign Studies University, 2015.

[18] M. Davies, British National Corpus. Oxford: Oxford University Press, 2014.

[19] G. Mazzon, A History of English Negation. Harlow: Longman, 2004.

[20] J. Zhang, Problems about Chinese Grammar. Beijing: China Social Science Press, 1987.

[21] X. Ding, "Similarities and differences of order/sequence of first and second language acquisition and its causes of formation". Applied Linguistics, 2006. 